\title{
EVIDENCIAS ARQUEOLÓGICAS EN LA AVENIDA CALLE REAL, VILCASHUAMÁN-AYACUCHO
}

\author{
Yuri I. CaVero Palomino \\ Universidad Nacional de Educación EnRIQUe Guamán y VALLE - La CANTUTA \\ david_aguilarb@yahoo.com
}

\section{RESUMEN}

En el presente artículo se exponen las evidencias arqueológicas consistentes en dos segmentos de canales y 2 restos de secciones de calzadas empedradas, halladas durante los trabajos de sustitución de la capa del pavimento y de las tuberías de agua potable y desagüe efectuadas en la primera cuadra de la Av. Camino Real en la ciudad de Vilcashuamán, la cual durante la época inka fue la capital del centro administrativo del mismo nombre.

Palabras Clave: Vilcashuamán, canales, calzada empedrada, gentil acequia, centro administrativo.

\begin{abstract}
In this article the archaeological evidence consisting of two segments of channels and 2 sections of cobbled remains of roads, found during work to replace the pavement layer and the water pipes and drainage made in the first block of exposed Av. Camino Real in the city of Vilcashuamán, which during Inca times was the capital of the administrative center of the same name.
\end{abstract}

KEYWORDS: Vilcashuamán, channels, paved road, ditch gentle, administrative center. 


\section{INTRODUCCIÓN}

En el presente artículo presentamos las evidencias de 2 secciones de canales y restos de 2 segmentos de calzada empedrada del Camino Inca halladas en el subsuelo de la 1ra cuadra de la Av. Camino Real en la ciudad de Vilcashuamán, Ayacucho. Estos hallazgos se efectuaron el año 2012, durante la ejecución del Monitoreo Arqueológico sobre infraestructura preexistente del proyecto: "REHABILITACION DE PISTAS Y VEREDAS DEL CERCADO DE VILCASHUAMÁN, PROVINCIA DE VILCASHUAMÁN AYACUCHO" (Código SNIP 180804), ejecutado por la Municipalidad Provincial de Vilcashuamán y fue autorizado mediante la RDR Nro 022-2012-DRC-AYA/MC.

\section{LA ACTUAL CIUDAD DE VILCASHUAMÁN}

La actual ciudad de Vilcashuamán es la capital de la provinvia del mismo nombre y se encuentra a $3450 \mathrm{msnm}$ y aproximadamente a $112 \mathrm{~km}$ hacia el sureste de la ciudad de Ayacucho y fue edificada sobre las estructuras arquitectionicas de lo que fue la ciudad inka. La provincia de Vilcashuaman se encuentra conformada por los distritos de Vischongo, Concepción, Vilcashuamán, Huambalpa, Carhuanca, Saurama, Acomarca e Independencia, con más de 100 anexos. Abarca un territorio de $1178.16 \mathrm{~km}^{2}$, con terrenos con altitudes que oscilan entre los $2500 \mathrm{msnm}$ y los $3880 \mathrm{msnm}$. Limita por el Sur con los distritos de Huambalpa y Saurama; hacia el Este: con el río Pampas; por el Oeste: con el río Vischongo y por el Norte: con el distrito de Concepción. La temperatura anual promedio es 14.6 ${ }^{\circ} \mathrm{C}$ y las precipitaciones anuales llegan a los $844.8 \mathrm{Mm}$. con intensidades pluviales entre Noviembre a Marzo y el resto del año las temperaturas mínimas son de $0.0 \mathrm{Mm}$ y la humedad relativa promedio es de $59 \%$.

Las formaciones geomorfológicas son variadas y pertenecen al Terciario - Cuaternario, existen rocas volcánicas del Cenozoico de la cordillera Occidental, donde confluyen indudablemente acciones de las fuerzas internas y externas que dan origen a variadas y complejas formas de relieves, desde valles encajonados hasta mesetas alto andinas. Los terrenos adyacentes a la ciudad de Vilcashuamán pertenecen a la región Quechua $(2300$ - $3500 \mathrm{msnm})$, que se extienden desde la confluencia del río Vischongo - Pampas hasta las inmediaciones del cerro Pillucho. Su topografía está formada por cerros de suave declive y cortado por quebradas y despeñaderos mesófilos. La región Suni (3500 - 4000 $\mathrm{msnm}$ ) presenta una topografía caracterizada por la presencia de bruscas ascensiones de acantilados, pañolerías y cerros elevados como Amaruqasa y Qatuncorralniyoq.

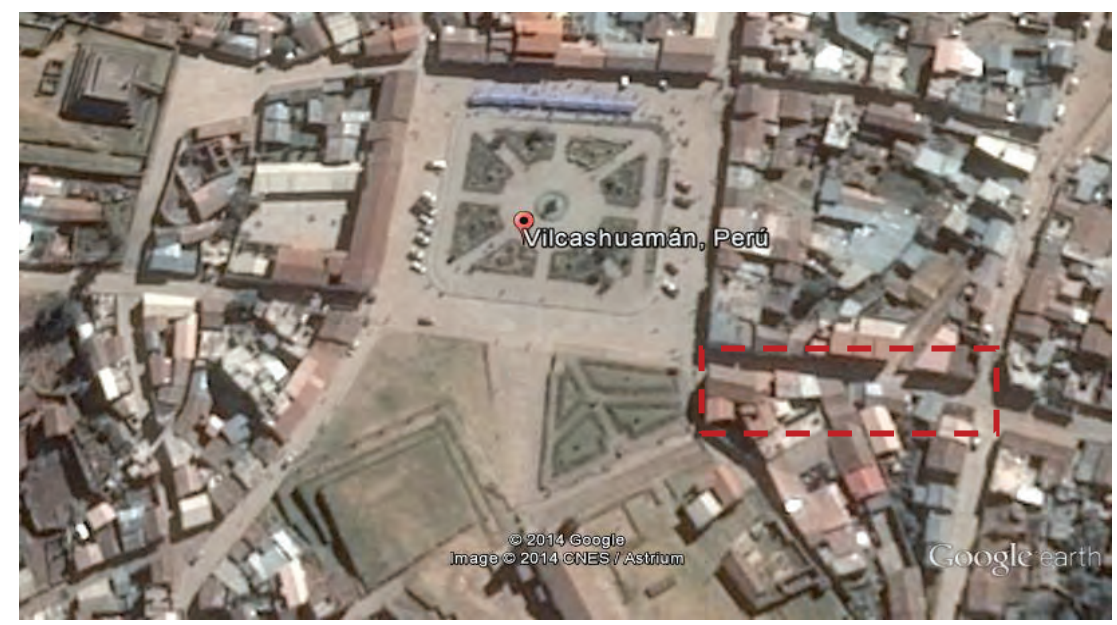

Figura 1: Ubicación de la primera cuadra de la Av. Camino Real en la Ciudad de Vilcashuamán (Modificado en base a la imagen satelital de: www.Googleearth.com) 


\section{LA CIUDAD INKA DE VILCASHUAMÁN Y EL HORIZONTE TARDIO:}

Vilcashuamán fue el primer centro administrativo, fundado y mandado edificar por Pachacuteq, luego de la derrota de los Chankas. Tenía como sede la actual ciudad de Vilcashuamán. El nombre mencionado por diferentes cronistas para referirse a este centro administrativo, provendría de la palabra quechua Willkawaman, la misma que derivaría de dos topónimos quechuas: Willca que significa: sagrado, adoratorio, viejo (en Aymara significa sol) y, Waman que significa Halcón. De la unión de estas dos palabras tendríamos: halcón sagrado, viejo halcón o adoratorio del halcón. Por lo cual, pensamos que se trataba de un territorio con un paisaje sagrado de mucha importancia desde Periodo Formativo, debido a la presencia en este territorio del centro ceremonial de Campanayuq Rumi, el cual podría haber sido la "guaca del viejo halcón sagrado".

Un dato Etnohistórico que reforzaría nuestra propuesta viene a ser la descripción que efectúa el cronista Juan Santa Cruz Pachacuti Yanque Salcamayhua, quien menciona que: “... Pachacutec Inga Yupanqui llegó con cuarenta mil indios de guerra a Vilcashuamán donde encontró siete guacas y demonios en figuras de curacas muy grandes, negros y muy feos eran llamados Aysavillca, Pariacaca, Chinchaycocha, Vallallo, Chuquivacra y otros dos Cañaris...". Al respecto debemos tener en cuenta que los trabajos arqueológicos efectuados en Campanayuq Rumi por Matsumoto y Cavero (2010), Matsumoto, Nesbit, Cavero y Mendoza (en prensa) han confirmado la presencia de diversos grupos cerámicos del periodo formativo proveniente precisamente de algunos de los territorios donde se ubican las guacas mencionadas por el referido cronista.

Los centros administrativos eran considerados como "Otros Cusco" y el cronista Guamán Poma de Ayala (Ibid) incluye dentro de ellos a: Quito, Tomebamba, Huánuco Pampa, Hatun Colla y Charcas; mientras que Pedro Cieza de León indica que las cabeceras de provincias eran: Vilcas, Xauxa, Bombón (Pumpu), Caxamalca, Guancabamba, Tomebamba, Latacunga, Quito, Caranqui, Hatuncana, Hatuncolla, Ayaviri, Chuquiabo, Chucuito. Para el caso de Vilcashuamán, Damián de la Bandera (1968:506) menciona que el Tucuyricoq que estaba a cargo de la administración de esta provincia: “... gobernaba cincuenta leguas de tierra, desde Uranmarca ques de aquel cabo de Vilcas seis leguas, hasta Acos, ques junto al valle de Jauja...”. De otro lado, el cronista Pedro de Carbajal refiere que: “... existió en el lugar una guarnición militar de treinta mil soldados y un acllawasi que albergaba a más de mil mujeres dedicadas al culto..." (1881:167).

Cieza de León (1967:159) destaca la importancia de los edificios que habían en ella y su ubicación "justo a la mitad" (-Chaupi-) del camino entre Quito y Santiago, menciona que Pachacutec fue el que ordenó su construcción y describe que: “ Los de la comarca, como supieron su estada allí, muchos vinieron a le ver haciéndole grandes servicios y firmaron con él amistad y por su mandato comenzaron a hacer aposentos y edificios grandes en lo que ágora llamamos Vilcas, quedando maestros del Cuzco para dar la traza y mostrar con la manera que habían de poner las piedras y losas en el edificio... ".

Los centros administrativos se caracterizaron por haber sido diseñados repitiendo la imagen física de la ciudad sagrada del Cusco; por ello, en Vilcashuamán se encuentra la plaza de forma trapezoidal (que podía albergar hasta 20,000 personas) y a hacia el lado Noroeste se edificó el Ushnu y el palacio del Inka, hacia el lado sur se construyeron el templo del sol y la luna, el acllawasi y otros edificios menores. Mientras las colcas fueron edificadas en las laderas del cerro Lawirasqa. Existía una red vial que lo articulaba con el Cuzco y con los otros pueblos que se encontraban bajo su jurisdicción (Cavero en prensa). Asimismo, algunos cronistas mencionan que al medio de esta plaza se hallaba un altar o escaño a manera de teatro, donde el señor se asentaba para ver los bailes y fiestas, asimismo, se encontraba una piedra del sacrificio y por el medio de esta gran plaza discurría una gentil y muy elaborada acequia. 


\section{ESTUdIOS ARQUEOLÓGICOS E HISTÓRICOS EFECTUADOS EN VILCASHUAMÁN}

La Ciudad Inca de Vilcashuamán ha sido descrita por diversos cronistas del siglo XVI-XVII, entre ellos: Joan de Santa Cruz Pachacuti Yanqui Salcamayhua, Pedro Cieza de León, Cabello de Balboa, Bernabé Cobo, Pedro de Carvajal, Antonio Vásquez Espinosa, entre otros. En los siglos XVIII-XIX, algunos aventureros y viajeros extranjeros describen a Vilcashuamán, entre estos destacan Leonce Angrand (1849), E. George Squier (1863-1865), Charles Wiener (1880), E. Middenford (1893), entre otros. A partir de los años 1900 serán algunos estudiosos peruanos que hacen referencia al sitio arqueológico, entre ellos José de la Riva Agüero (1995), Jose Ruiz Fowler (1924), Alberto Arca Parró (1927), Pio Max Medina (1930), Victor Von Hagen (1969), Agusto Paz Soldán (1960), Fernando Belaunde Terry (19631965), entre otros.

En cuanto se refiere a los trabajos de carácter arqueológicos, podemos mencionar los desarrollados por: Carlos Guzmán Ladrón de Guevara (1959), quien efectúa un reconocimiento arqueológico en los territorios de Vilcashuamán. El año 1959 Luis Guillermo Lumbreras presenta una ponencia en el II Congreso Nacional de Historia del Perú, donde menciona los sitios Chankas de: Aya Orqo, Arqalla, Chaupi Moqo, Patarajay, Ayapata, Willca Chanca, Ticllarasu, Aya Taqui, Pillucho, Lawirasqa, estableciendo la cerámica Arqalla como distintivo cultural Chanka.

En 1962, el Arquitecto Fernando Belaunde Terri, publica una revista en la que refiere: "Vilcashuamán no ha muerto a pesar de haber perdido esa jerarquía política. No es el cadáver de una urbe, como Huánuco Viejo y Marcahuamachuco. Ha sobrevivido a los cambios políticos pero ya no es la gran "cabeza de región" sino que languidece en su aislamiento en la rustica y humilde vida pueblerina del villorrio andino" (Belaunde, 1962:51).

El año 1966 y 1967 Carlos Chaud realiza investigaciones arqueológicas en Vilcashuamán, como resultado de los cuales menciona que los primeros habitantes en Vilcashuamán fueron los Chankas. En 1969, Jorge Torres Palomino realiza investigaciones arqueológicas en Pillucho y reconocimientos superficiales en los sitios de Qollqa Horno, Qollqa Moqo, China Qasa y Raqchipata. Estableciendo evidencias de la presencia de la cultura Chanka en estos sitios así como en Pillucho.

En 1977, el Ministerio de Industria y Turismo (MIT) y el Instituto Nacional de Cultura (INC), bajo la dirección de Miguel Pazos y Santiago Agurto suscribieron convenios para efectuar estudios y proyectos de ejecución de obras, destinados a la puesta en valor y su habilitación parcial para el turismo.

A partir de 1977-1980, Enrique Gonzáles Carré, Jorge Cosmópolis y Jorge Lévano continuaran realizando investigaciones, producto del cual posteriormente publican en 1982 el libro titulado "La Ciudad Inca de Vilcashuamán".

En 1977, Graziano Gasparini y Luise Margorlies, como parte de sus investigaciones menciona al centro administrativo Inka de Vilcashuamán y desarrollan reconstrucciones hipotéticas del sitio arqueológico, en base a los dibujos de L. Angrad, y el plano realizado por Ch. Wiener. En 1979, Lorenzo Huertas Vallejo, publica un artículo, que refiere sobre la revisita realizada a Vilcashuamán en 1729; este documento registra: la condición social de los centros poblados (pueblos, haciendas, obrajes, y ayllus); la diversidad étnica y, el problema del poder local (como de los curacas, alcaldes y cobradores), lo más interesante se registra el aspecto económico de la población activa y la tributación en el año 1729.

Ese mismo año (1979), Noé Jave Calderón realiza una investigación cronística y etnohistoria de las Qollqas de Lawirasqa, para lo cual se centra en el estudio del documento de petición de tierra realizada por Don Diego de Guillen de Mendoza "De las cuadras y tierras de Vilcas, Molino y Obraje, del año 1586"; en el cual hace apreciaciones sobre los sistema de almacenamiento Inka, concentrándose en el estudio arqueológico y etnohistórico. 
En 1983 Hugo Ludeña publica un artículo sobre Vilcashuamán, en el hace un resumen del proceso prehispánico de Vilcashuamán, problemas de propiedad durante la colonia; de igual manera trata de sintetizar la información arqueológica existente hasta ese entonces y también hace referencia sobre el mal estado de conservación del sitio arqueológico.

A inicios de los años 1982-1984 Manuel Cuentas y A. Astete (1985) efectúan trabajos de excavaciónes arqueológicas, conservación y restauración en el Ushnu, kallanka y depósitos reales. Estos trabajos estuvieron orientados exclusivamente a rescatar y conservar estos monumentos de factura inca, el financiamiento estuvo a cargo de la CORFA y mediante un convenio con el INC filial Ayacucho. En 1995 el Consejo Transitorio de Administración Regional "Los Libertadores Wari” en convenio con el INC departamental de Ayacucho realiza nuevamente actividades de Restauración de sectores que no se habían intervenido durante los años 1982-1984 en el Templo del Sol y la Luna. Posteriormente en año 1996 el Consejo Transitorio de Administración Regional "Los Libertadores Wari” firman un convenio con la UNSCH para la ejecución de labores de Rehabilitación y Conservación de los sectores del Templo el Sol, Ushno y Piedra del sacrificio, los cuales fueron considerados como parte de las actividades programadas para el desarrollo y promoción el turismo en la región de Ayacucho.

En 1997 el CTAR “Los Libertadores Wari” en convenio con la UNSCH continúan ejecutando trabajos de rehabilitación y conservación de monumentos arqueológicos, tales como el Templo el Sol en Vilcashuamán y el conjunto arqueológico de Pumaccocha en Vischongo. Luego en 1999, el CTAR "Los Libertadores Wari" se construye el museo en convenio con el INC-Ayacucho y se adquirió un terreno ubicado al ingreso de la ciudad de Vilcashuamán.

El año 2002 Ulises Larrea y Yuri Cavero, hacen una evaluación Arqueológica hacia el lado Este de la ciudad de Vilcashuamán, refiriendo la existencia del Centro Ceremonial en forma de "U" llamado Campanayuq Rumi y que corresponde al Periodo Formativo. A Partir del año 2004 el Instituto Nacional de Cultura, a través del proyecto Qhapaq ñan inició una intervención multidisciplinaria en la provincia de Vilcashuamán, el mismo que incluyó varios componentes, destacando el de patrimonio colonial y republicano, etnográfico, geográfico, prevención y defensa del patrimonio, museológico y arqueológico. Los trabajos mencionados se desarrollaron con el objetivo de preservar y conservar el Patrimonio Cultural de la Nación, el ordenamiento territorial y la puesta en valor del sitio arqueológico, con la participación activa de las comunidades locales

A partir del año el 2007, Yuri Cavero y Yuichi Matsumoto, vienen desarrollando investigaciones arqueológicas en Campanayuq Rumi, habiendo, definido la presencia de una ocupación muy densa durante el periodo Inicial y Formativo. Los resultados preliminares indican que es necesario considerar a la región de Vilcashuamán, como un territorio de suma importancia en el desarrollo histórico de los Andes Centrales desde el Periodo Inicial y Formativo.

El 2007 Ismael Pérez y otros, publican el libro titulado "Vilcashuamán, Paisaje, Historia y Cultura", haciendo un recuento de las excavaciónes y trabajos de restauración efectuados entre los años 19961998 por Ismael Pérez en el Ushnu y Templo del Sol y La Luna. El año 2009 por Elizabeth Ochoa Zea, bajo la asesoría de Yuri Cavero Palomino presenta y sustenta su tesis de bachillerato en la Universidad Nacional de San Cristóbal de Huamanga titulado: "Investigaciones arqueológicas en Waychawaqana, Vilcashuamán, Ayacucho".

El año 2010 el Bachiller Edison Mendoza, bajo la asesoría de Yuri Cavero presenta a la UNSCH su tesis de licenciatura titulado "Contextos Rituales Tardíos, en el Centro Ceremonial Formativo de Campanayuq Rumi, Vilcashuamán-Ayacucho". Ese mismo año, los estudiantes Ronald Sulca, Jhon Rene Huamani, Vilma Quispe y Maribel Solier, Efectúan análisis de los materiales arqueológicos de otros sectores del mismo sitio arqueológico y presentan sus tesis de bachillerato ante la Universidad Nacional de San Cristóbal de Huamanga. 
El año 2012 el Dr. Idilio Santillana publica el libro titulado: "Vilcas Huamán: Paisaje sagrado y territorio", el cual viene a ser la traducción de su tesis doctoral; describe los principales restos arqueológicos que se encuentran en la zona de Vilcashuamán y hace una interpretación del paisaje y su relación con la ideología andina. Incluye únicamente datos hasta el año 2000.

El año 2012, Yuri Cavero efectúa un monitoreo arqueológico para las obras de cambio de sistemas de tuberías de agua potable y desagüe en la primera cuadra de la Av. Camino Real, a cargo de la Municipalidad Provincial de Vilcashuamán, hallando los restos de dos canales incas muy bien elaborados, uno de los cuales se dirige hacia la parte baja del Templo del Sol y la Luna, mientras el otro hacia la gran plaza inca de Vilcashuamán, asimismo se encontraron dos segmentos de la calzada empedrada del camino Inka.

\section{FASES DE EJECUCIÓN DEL TRABAJO ARQUEOLÓGICO:}

\section{CHARLAS DE INDUCCIÓN Y CAPACITACIÓN:}

Como medida preventiva, se efectuó charlas de inducción para el personal que estuvo a cargo de la ejecución de la obra y para los vecinos de las calles involucradas en el presente proyecto. Los temas tratados estuvieron relacionados a tomar conciencia sobre la necesidad de protección del Patrimonio Arqueológico e Histórico de la Nación.

\section{2.- EVALUACIÓN Y REGISTRO DE LOS PERFILES:}

Esta fase del trabajo se desarrolló durante la reapertura de las zanjas para la sustitución de las tuberías de agua potable y desagüe. La evaluación, estudio y registro de los perfiles de las zanjas reaperturadas permitió ubicar en la primera cuadra de la Av. Camino Real, dos secciones de restos de calzada empedrada, así como el hallazgo de secciones de dos imponentes canales Inkas, los mismos que han sido debidamente registrados y tratados a fin de garantizar su conservación y en parte, su exhibición a la población y a los turistas
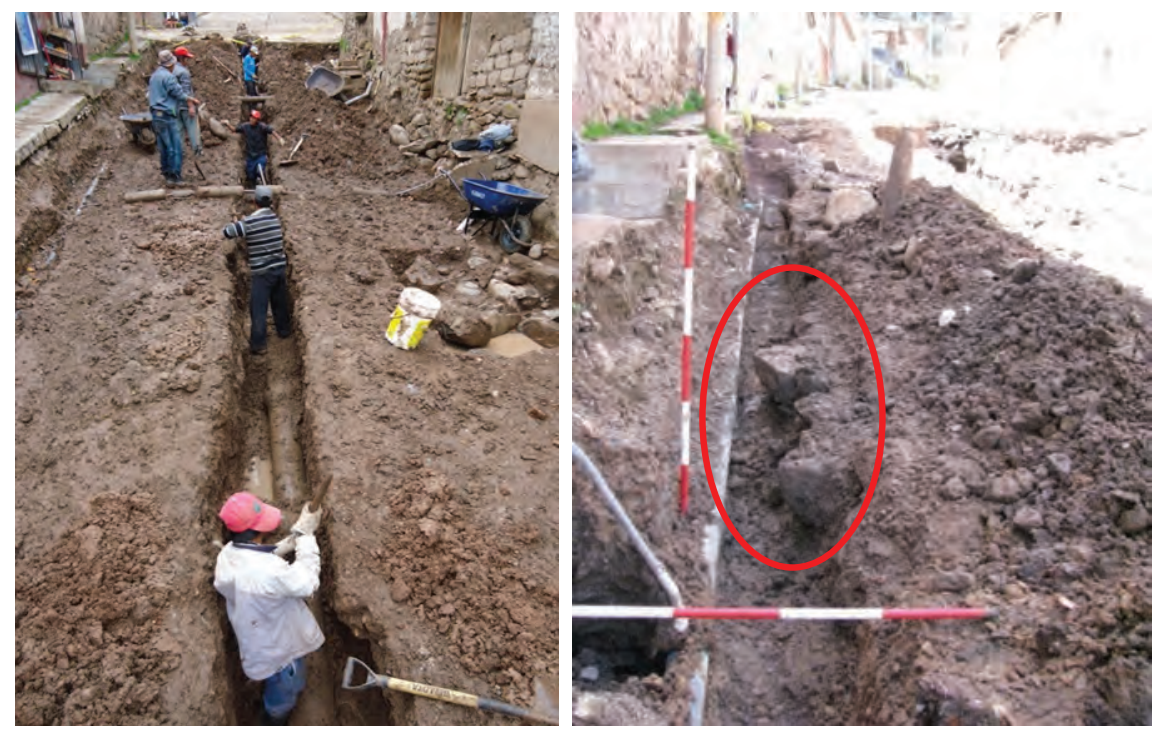

Figuras 2 y 3: Proceso de reapertura de las zanja de tubería de agua potable y desagüe en la parte media de la vía; a la derecha obsérvese los restos de la calzada empedrada que son visible en una sección del perfil. 


\section{3.- PLAN DE CONTINGENCIA DESARROLLADO FRENTE A HALLAZGOS ARQUEOLÓGICOS FORTUITOS:}

Como se ha referido anteriormente, durante la ejecución de las obras, se han logrado ubicar restos arqueológicos en la primera cuadra de la Av. Camino Real, por lo cual, se procedió a su registro y protección de los mismos. Para cuyo efecto se efectuó las coordinaciones del caso con la Dirección Regional de Cultura de Ayacucho.

\section{4.- REGISTRO DE EVIDENCIAS ARQUEOLÓGICAS:}

Esta labor se desarrolló siguiendo los lineamientos metodológicos y técnicos propios de la arqueología, los cuales se basaron en la demarcación y excavación de 4 unidades de excavación, 1 unidad de 1m x $1 \mathrm{~m}$ (Unidad 1); 1 unidad de $2 \mathrm{~m} \times 1 \mathrm{~m}$ (Unidad 2) y 2 unidades a modo de trinchera de $1.50 \mathrm{~m} \times 5 \mathrm{~m}$ (Unidades 3 y 4). Las características y secuencia estratigráfica hallada en cada una de las unidades aperturadas son descritas en las siguientes líneas:

\section{UNIDAD DE EXCAVACIÓN NRO 1}

Dimensión: $1 \mathrm{~m} \times 1 \mathrm{~m}$.

Ubicación: Lado Este de la zanja de tubería de desagüe.

Secuencia estratigráfica: La secuencia estratigráfica hallada es la siguiente:

Capa A: Viene a ser la capa de asfalto que cubre toda la superficie del terreno. Posee un espesor que varía entre $16 \mathrm{~cm}$ a $18 \mathrm{~cm}$. No hay evidencias de material arqueológico.

Capa B: Material de relleno conformado por tierra de color marrón claro, cascajo y cantos rodados pequeños. Presenta un espesor de $30 \mathrm{a} 60 \mathrm{~cm}$, se trata de una capa de origen moderno dispuesto en el año 1980 para nivelar el terreno sobre el cual se instaló la capa de asfalto (Capa A). En su interior se observa la presencia de material y basura moderna, destacando la presencia de clavos, pilas, plásticos y vidrio. No se hallaron restos arqueológicos.

Capa C: Tierra arcillosa de color marrón oscuro, de contextura suelta y suave, en cuya parte media y superficial se observan algunas piedras planas distribuidas de manera uniforme a una profundidad de $90 \mathrm{~cm}$ debajo del nivel de la pista. Los cuales al ser limpiados resultaron ser restos de un empedrado que podría corresponder al camino inca. A la altura de la mitad de la vía se observa las huellas de la antigua zanja por donde discurren las tuberías del desagüe y hacia el lado izquierdo de la vía, se observa las huellas de otra zanja de menor ancho, que corresponde al lugar por donde discurren las tuberías del abastecimiento del agua potable de las viviendas de la avenida. 

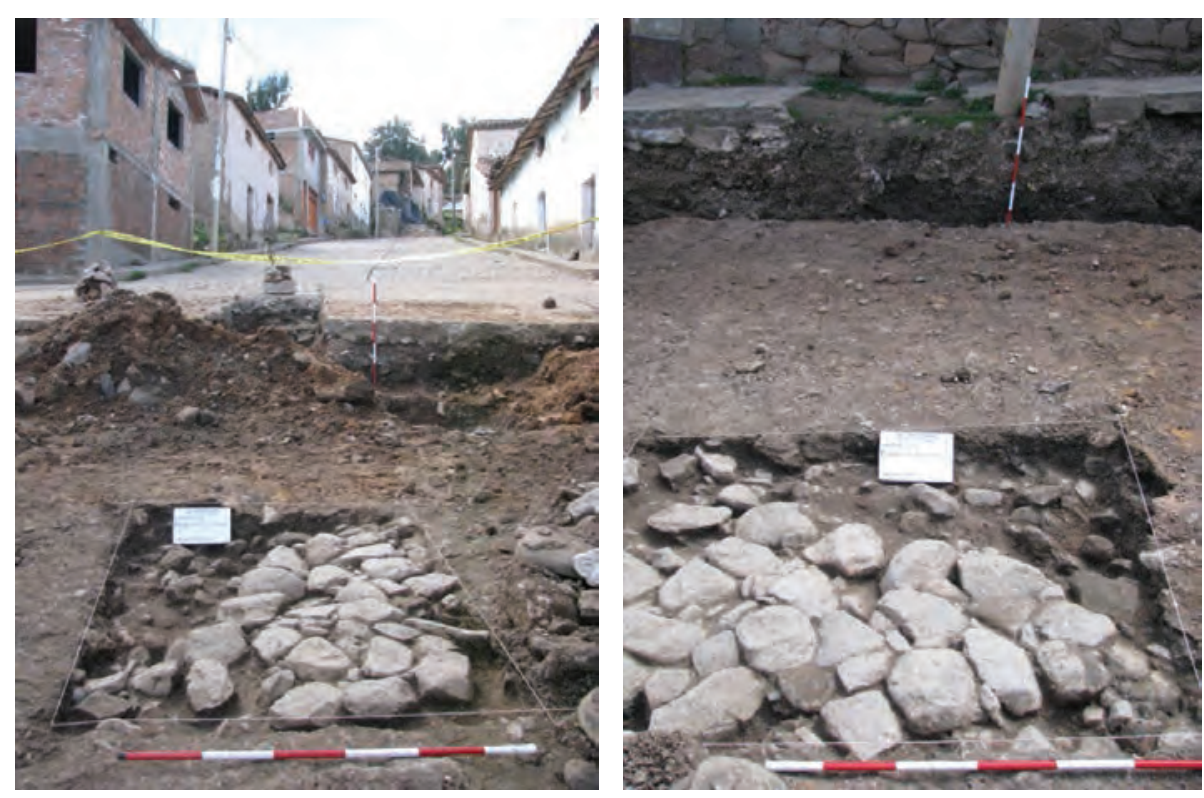

Figuras 4 y 5: Unidad 1, Restos de empedrado hallados a $90 \mathrm{~cm}$ de profundidad del nivel de la pista. Correspondería a la calzada del Qhapaq Ñan que de la plaza Inka salía en dirección hacia el Cuzco.

\section{UNIDAD DE EXCAVACIÓN NRO 2}

\section{Dimensión: $2 \mathrm{~m} \times 1 \mathrm{~m}$.}

Ubicación: Lado Noreste de la 1ra cuadra de Av. Camino Real.

Secuencia estratigráfica: La secuencia estratigráfica hallada es la siguiente:

Capa A: Viene a ser la capa de asfalto que cubre toda la superficie del terreno. Posee un espesor que varía entre $18 \mathrm{~cm}$ a $20 \mathrm{~cm}$. No hay evidencias de material arqueológico.

Capa B: Material de relleno conformado por tierra de color marrón claro, cascajo y cantos rodados de tamaños variados. Presenta un espesor de 40 a $60 \mathrm{~cm}$, se trata de una capa de origen moderno dispuesto para nivelar el terreno sobre el cual se instaló la capa de asfalto (Capa A). En su interior se observa la presencia de secciones de tubos de PVC y basura moderna, destacando la presencia de clavos, pilas, plásticos y vidrio. En el lado sur se observa el alineamiento de 3 piedras planas dispuestas en forma horizontal. No se hallaron materiales arqueológicos.

Capa C: Tierra arcillosa de color marrón oscuro, de contextura suelta y suave, a una profundidad de $75 \mathrm{~cm}$ del nivel del piso de cemento se observa la presencia de piedras y cantos rodados planos distribuidos de manera uniforme en toda la extensión de la unidad. La limpieza develó la presencia de una sección de empedrado, el cual se asemeja al hallado en la unidad 1 y ambos corresponderían a restos de la calzada del camino inca que discurría por dicha calle. Hacia el lado izquierdo, se observa las huellas de la zanja cavada el año 1980 el cual posee aproximadamente $40 \mathrm{~cm}$ de ancho, en cuyo interior se encuentran las tuberías de PVC del sistema de abastecimiento de agua potable. 


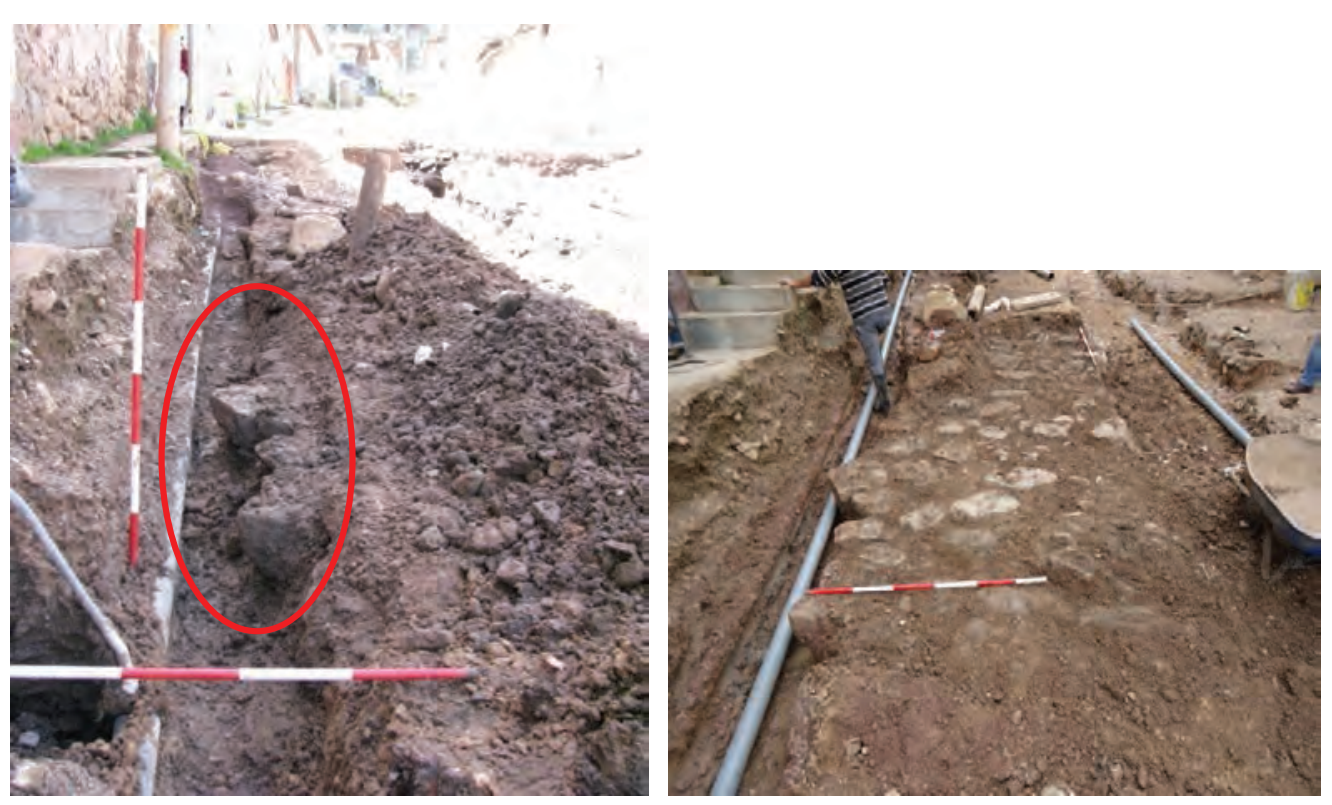

Figuras 6 y 7: Secuencia fotográfica que muestra la ubicación y limpieza de restos de empedrado que corresponderían a la calzada empedrada del camino Inka.

\section{UNIDAD DE EXCAVACIÓN NRO 3}

Dimensión: $1.5 \mathrm{~m}$ x $5 \mathrm{~m}$.

Ubicación: Lado medio de la 1ra cuadra de Av. Camino Real.

Secuencia estratigráfica: La secuencia estratigráfica hallada es la siguiente:

Capa A: Viene a ser la capa de asfalto que cubre toda la superficie del terreno. Posee un espesor que varía entre $17 \mathrm{~cm}$ a $20 \mathrm{~cm}$. No hay evidencias de material arqueológico.

Capa B: Material de relleno conformado por tierra de color marrón claro, cascajo y cantos rodados de tamaños variados. Presenta un espesor de 30 a $45 \mathrm{~cm}$, se trata de una capa de origen moderno dispuesto para nivelar el terreno sobre el cual se instaló la capa de asfalto (Capa A). En su interior se observa la presencia de material y basura moderna y no contiene restos arqueológicos. Casi al finalizar esta capa se observa que existen diversas tuberías de cemento en mal estado, que corresponden al antiguo sistema de abastecimiento de agua domiciliaria de las viviendas.

Capa C: Tierra arcillosa de color marrón oscuro, de contextura suelta y suave. En la parte media en los perfiles de la zanja reaperturada se observan algunas piedras labradas distribuidas de manera uniforme en forma vertical. Los cuales al ser limpiados resultaron ser restos de los muros laterales de un canal Inka (canal 1), el mismo que discurre en forma perpendicular y oblicua en relación al eje central de la vía y en dirección Sureste-Noroeste. El canal referido se encuentra demarcado por dos muros de una sola cara, las cuales se caracterizan por haber sido construidas por piedras debidamente talladas y perfectamente encajadas con mortero de arcilla, tiene una altura conservada variable de 40 a $60 \mathrm{~cm}$ de alto y su piso se encuentra debidamente enlajado por bloques de piedras planas de formas alargadas y unidos con mortero de arcilla de color rojo. Se encuentra alterado en su parte media, a causa de la presencia de la zanja del antiguo sistema de desagüe y en la parte sur por la presencia de un canal de drenaje que discurre en forma paralela al eje de la vía y fue construido en época moderna. 
Se puede concluir que en la década de los años 80 , al haberse cavado dichas zanjas, se han cortado algunas secciones de los muros del canal, y en otros casos las conexiones domiciliarias han sido instaladas sobre la parte superior del muro del canal inca.

Capa D: Material compuesto por arena y gravilla que se encuentra en la parte interna del canal. Esta capa se caracteriza por ser de color marrón muy oscuro y por partes inclusive llega a poseer un color negro y húmedo, en su interior se encontró algunos fragmentos de cerámica, fragmentos de huesos de camélidos y artefactos líticos aislados.
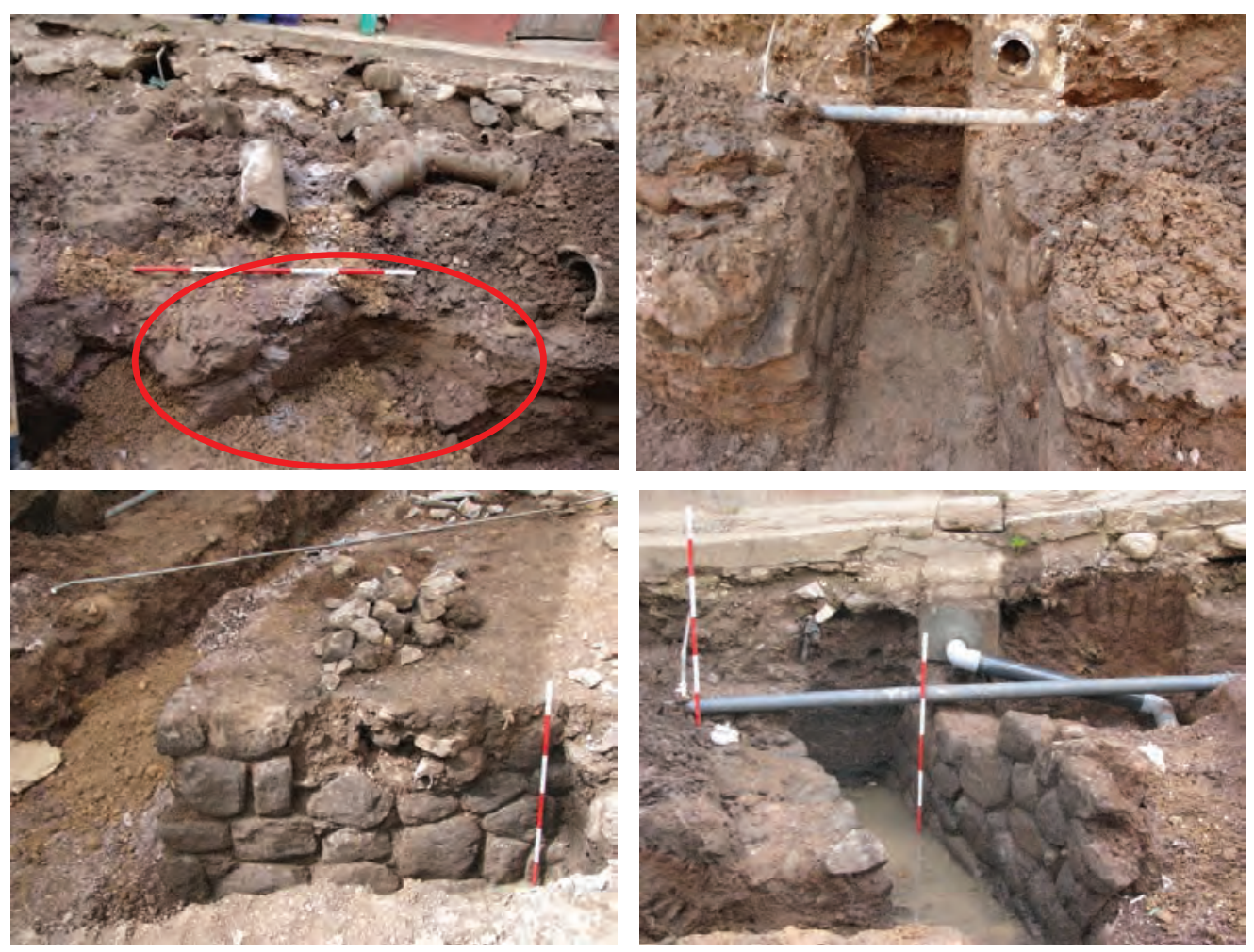

Figuras 8,9,10 y 11: Secuencia fotográfica de la ubicación y limpieza del canal Nro 1 


\section{UNIDAD DE EXCAVACIÓN NRO 4}

Dimensión: $1.5 \mathrm{~m} \times 5 \mathrm{~m}$.

Ubicación: Lado Oeste de la 1ra cuadra de Av. Camino Real.

Secuencia estratigráfica: La secuencia estratigráfica hallada es la siguiente:

Capa A: Viene a ser la capa de asfalto que cubre toda la superficie del terreno. Posee un espesor que varía entre $15 \mathrm{~cm}$ a $20 \mathrm{~cm}$. No hay evidencias de material arqueológico.

Capa B: Material de relleno conformado por tierra de color marrón claro, cascajo y cantos rodados de tamaños variados. Presenta un espesor de 30 a $40 \mathrm{~cm}$, se trata de una capa de origen moderno dispuesto para nivelar el terreno sobre el cual se instaló la capa de asfalto (Capa A). En su interior hay presencia de material y basura moderna. No se encontraron restos arqueológicos; al finalizar la capa se observan diversas tuberías de cemento en mal estado, que corresponden al sistema de abastecimiento de agua potable domiciliaria de las viviendas ubicadas a ambos lados de la vía donde se ejecutó la obra.

Capa C: Tierra arcillosa de color marrón oscuro, de contextura suelta y suave. En los perfiles de la zanja ubicada hacia la parte media de la vía, se observan algunas piedras labradas distribuidas en forma vertical. Al ser limpiadas resultaron corresponder a los muros laterales de otro canal inca (canal 2), el mismo que discurre en dirección Noreste-Suroeste. Se encuentra demarcado por dos muros paralelos y de una sola cara, construidos por piedras debidamente talladas y perfectamente encajadas con mortero de arcilla, tiene una altura conservada de aproximadamente $45 \mathrm{a} 60 \mathrm{~cm}$ de alto y su piso se encuentra enlajada por piedras planas de formas alargadas y unidos con mortero de arcilla roja. Hacia el lado Noreste y debajo del cimiento de una vivienda moderna, se ubicó una sección del canal con su respectiva tapa "in situ", lo cual nos indicaría que todo este canal en la época inca se encontraba cubierto por lajas de piedras planas.

Al igual que el canal 1 , se encuentra alterado en su parte media por la zanja del antiguo sistema de desagüe y en la parte sur por la presencia de un canal moderno que discurre en forma paralela al eje de la vía. Asimismo, es visible la cantidad de tuberías de agua y desagüe que han alterado y destruido partes de los muros del canal inca Nro 2.

Capa D: Se consideró al material compuesto por arena y gravilla que se encuentra en la parte interna del canal. Esta capa se caracteriza por ser de color marrón muy oscuro y por partes inclusive llega a poseer un color negro y muy húmedo; al interior del cual se encuentran fragmentos de cerámica, fragmentos de huesos de camélidos y artefactos líticos, todos ellos sin contexto arqueológico definido. 

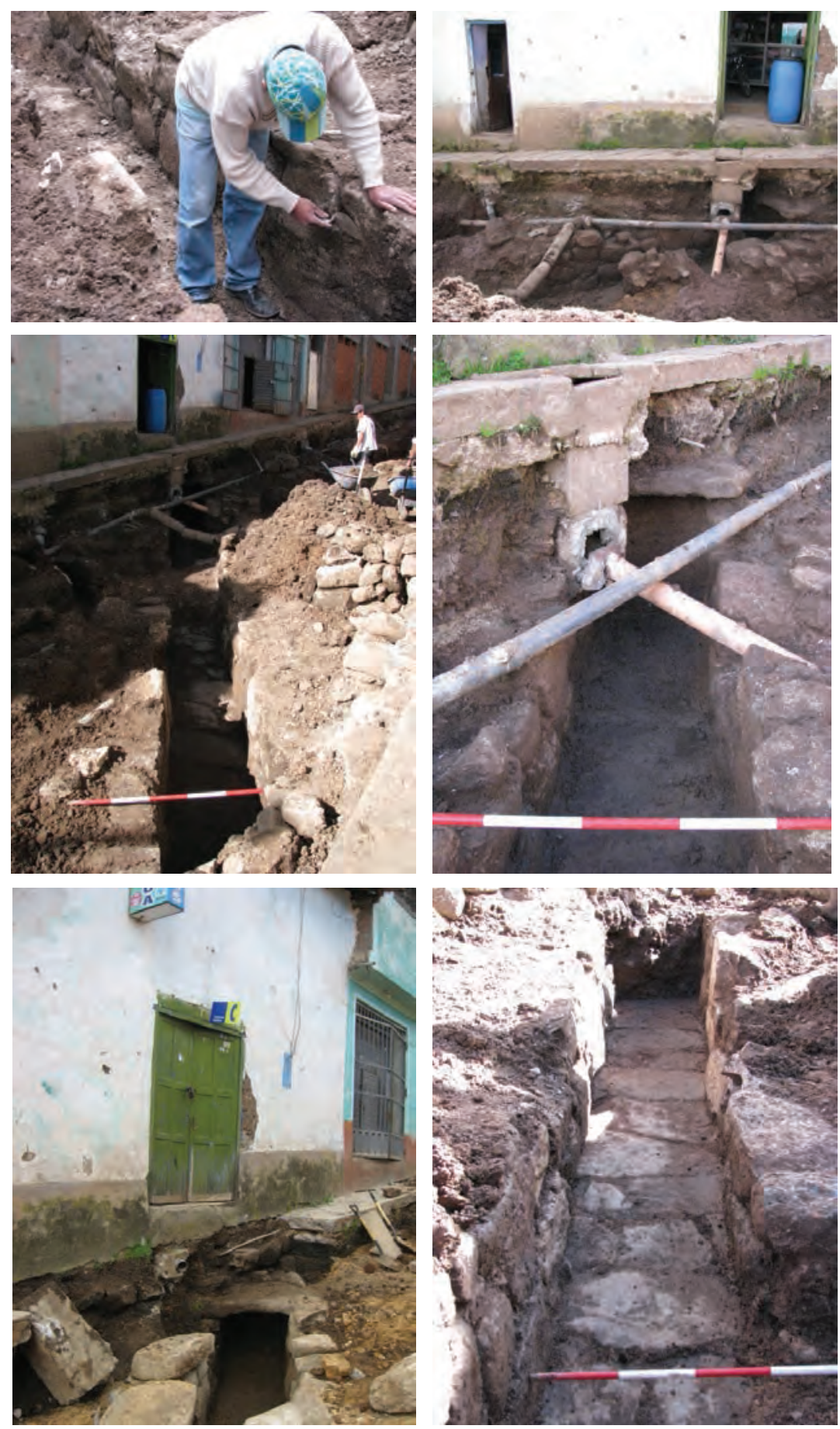

Figuras 12, 13, 14, 15, 16 y 17: Secuencia del proceso de limpieza del canal Inka Nro 2: obsérvese la laja del techo del canal que se encuentra debajo de la vereda y vivienda moderna. Detalle de las características y acabado que presenta el empedrado del piso del canal. 


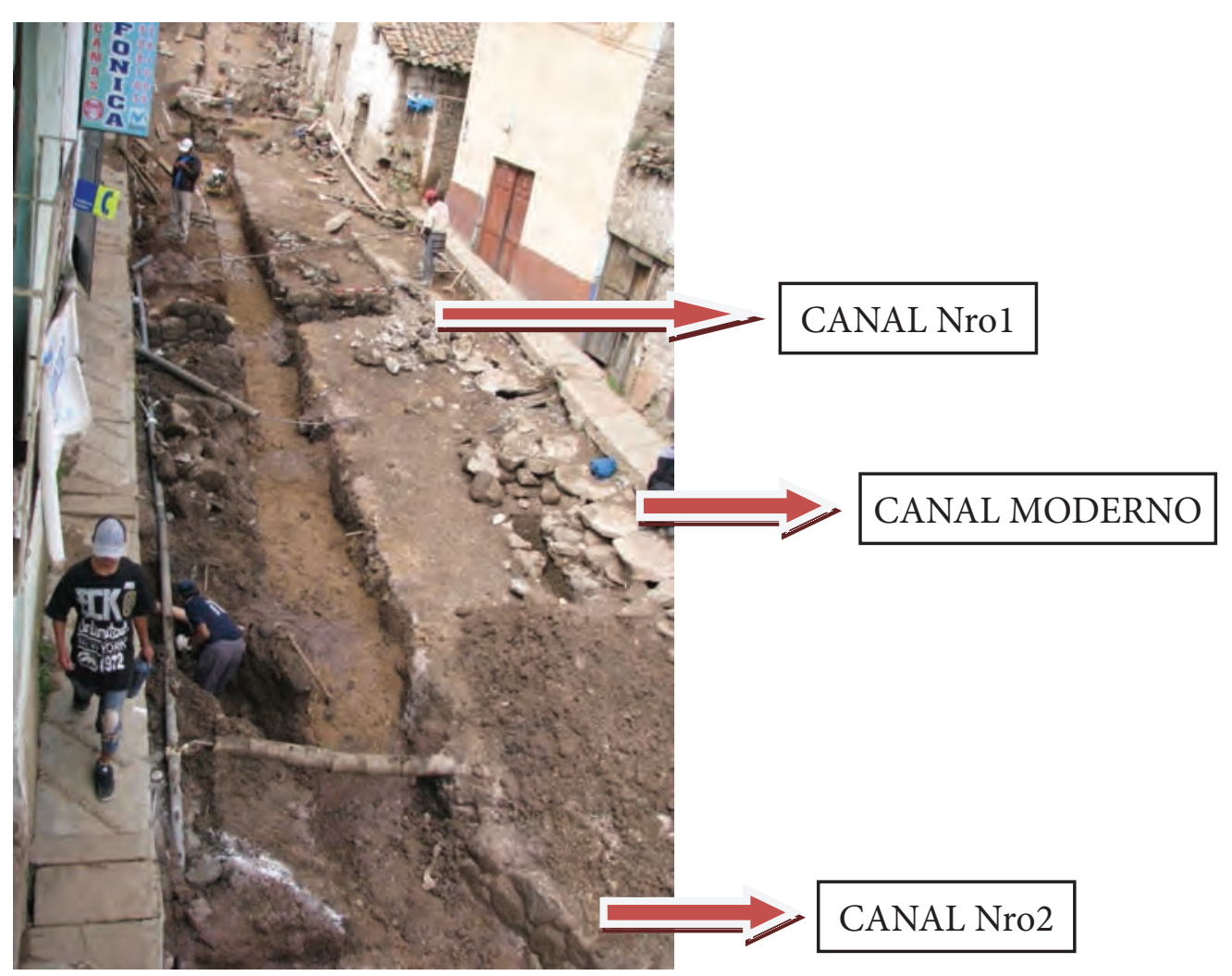

Figura 18: Vista Panorámica de la 1ra cuadra de Av. Camino Real y ubicación de los canales inkas hallados.

\section{PROTECCIÓN DE EVIDENCIAS ARQUEOLÓGICAS}

Debido al hallazgo de restos arqueológicos correspondientes a dos secciones de empedrados y restos de dos canales Inkas seccionados, fue necesario diseñar y seguir una estrategia de protección y conservación de los mismos. En el caso de las dos secciones de empedrados, estos fueron fotografiados, descritos y dibujados; debido a que se encuentran a una considerable profundidad en relación al nivel de la superficie de la vereda (entre 90 a $110 \mathrm{~cm}$ por debajo de ella), se optó por volver a cubrirlo con en su totalidad. Este procedimiento se hizo empleando tierra cernida, la misma que fue distribuida de manera uniforme por un espesor de $20 \mathrm{~cm}$ sobre el empedrado. Luego, encima de esta capa se dispuso otra capa de tierra y gravilla con un espesor de $15 \mathrm{~cm}$; finalmente, recién encima de esta capa descansa la capa de base del asfalto $(40 \mathrm{~cm})$ y finalmente la pista de cemento. El procedimiento antes descrito, permite garantizar la conservación total del empedrado hallado y a la vez, evita un contacto directo entre el resto arqueológico y la capa de material moderno que conforma la pista de cemento de la calle tratada.

En el caso de las secciones de canales Inkas hallados, las labores de conservación incluyeron primeramente la limpieza de las piedras del paramento de los muros; en segundo lugar, la instalación de un caping de protección de $20-30 \mathrm{~cm}$ de alto, construido con de piedras unidas con arcilla y barro sobre la parte superior de los muros de los canales; en tercer lugar, la instalación de una capa de tierra de $20 \mathrm{~cm}$ de espesor sobre la nueva cabecera de los muros. En cuarto lugar, a fin de darle mayor 
estabilidad y garantizar su protección e integridad, al interior del canal, se optó por construir pircas de piedras unidos con barro en distancias equidistantes, de modo que al interior de estos espacios se rellenó con tierra cernida; finalmente, sobre ella se distribuyó de manera uniforme una capa de gravilla y tierra por $20 \mathrm{~cm}$ de espesor, sobre el cual finalmente recién se instaló la capa de la base de la pista y sobre esta recién descansa el afirmado, el cual en los puntos donde se encuentran los canales tuvieron que ser previamente sujetados con un armazón de fierro y anclaje a fin de derivar el peso de la superficie hacia los lados laterales, de modo que los muros de los canales no se vean directamente afectados por el peso.

De otro lado, como parte de las medidas de protección y conservación de los restos arqueológicos hallados, en coordinación con la Municipalidad de Vilcashuamán se ha visto por conveniente cambiar el tipo de transito que deberá soportar la 1ra cuadra de la Av. Camino Real. Inicialmente, esta vía estaba concebida como una calle de tránsito vehicular pesado. Sin embargo, con la finalidad de proteger a los canales que se encuentran en el subsuelo, esta vía será únicamente de tránsito vehicular liviano y de un solo sentido; para cuyo efecto se procedió a darle mayor ancho $(1.50 \mathrm{~cm})$ a las veredas de ambos lados de la vía y se ubicará un panel de señalización que indique el tipo de tránsito vehicular liviano permitido. Finalmente, se decidió acondicionar dos secciones de los canales para que sean mostrados al público y al turismo. Para cuyo efecto estas 2 secciones de los canales han sido debidamente limpiadas, protegidos y acondicionados; incluyendo la instalación de dos sistemas de drenajes para la evacuación del agua del subsuelo.

\section{A MODO DE CONCLUSIONES}

Las evidencias arqueológicas halladas durante los trabajos de Monitoreo Arqueológico efectuado en la primera cuadra de la avenida Calle Real, nos demuestran que debajo de la actual ciudad de Vilcashuamán se encuentran aún bajo tierra diversas evidencias arqueológicas que requieren ser estudiados y registrados para un mejor conocimiento de la ocupación Inka y para precisar con mayores evidencias la distribución de los diferentes sectores o elementos que conformaban el núcleo urbano de la ciudad Inka y así compararlas con las informaciones etnohistóricas brindadas por los diversos cronistas.

El área donde se desarrolló el presente monitoreo (primera cuadra de la Av. Camino Real) se encontraría dentro del área conocida como la plaza inca, del cual el cronista Pedro de Carbajal menciona lo siguiente "Hay una plaza muy grande que pueden caber en ella muy bien más de veinte mil hombres, la cual mandó el inga hacer a mano, y cegó una laguna muy grande que allí había para este efecto". Asimismo, se describe que al medio de la plaza también existía una pila a modo de pequeño estrado donde se sacrificaban los animales y niños tiernos, cuyas sangre eran ofrecidos a los dioses.

En base a las evidencias arqueológicas halladas en la primera cuadra de la Av. Camino Real, podemos plantear que la plaza de Vilcashuamán hacia el lado Este llegaba únicamente hasta el punto donde se encuentran las dos secciones del muro Pachacuteq. En efecto, en base a la lectura y control detallado de los perfiles de las zanjas de agua potable y alcantarillado que fueron inicialmente cavados en los años 80 y reabiertos el 2012, podemos plantear que, los dos puntos donde se hallaron los restos de calzada empedrada corresponden a parte de la calzada empedrada del Qhapaq Ñan que desde la plaza Inka de Vilcashuamán salía en dirección al Cuzco. Las evidencias halladas se encuentran en un área que se encuentra en la parte externa del referido muro. De otro lado, las secciones de muros de los dos canales (canal 1 y canal 2) hallados en la parte media y baja de la misma avenida, se encuentran hacia el lado Suroeste del muro Pachacuteq y por ende al interior de lo que los cronistas llaman la gran plaza Inka. 
Al respecto Cieza de León (1551(1941: 266) refiere que “...Por medio de esta plaza pasaba una gentil acequia, traída con mucho primor, y tenían los señores sus baños secretos para ellos y sus mujeres." En base a los hallazgos efectuados sostenemos que las dos secciones de canales hallados en la primera cuadra de la Av. Camino Real, corresponderían a la "gentil acequia" descrita por Cieza. En el caso del Canal-1 este tiene una orientación Sureste-Noroeste y precisamente se dirige hacia la parte media de la plaza y este canal estaría dividiendo en dos sectores Hanan-Urin a la ciudad Inka. En lo que se refiere al canal-2, este se orienta en una dirección muy distinta al anterior, es decir, su orientación es de Noreste hacia Suroeste, lo cual implica que en algún punto no muy lejano del lugar donde se halló se une con el canal-1 o existe una fuente de almacenamiento de agua a donde llegan las aguas traídas por el canal 1 y luego son redistribuidas a distintos sectores. En el caso del canal-2 es importante señalar que hacia el Suroeste éste se proyecta hacia la parte baja del templo del Sol y la Luna.

Los trabajos desarrollados también han demostrado que el canal que discurre en forma paralela a la calle Camino Real y por el frente del Templo del Sol y La luna, no son de manufactura Inka, es en realidad un canal moderno, el cual ha sido hecho con material pétreo reutilizado pero con empleo de mortero de cemento e incluso en algunas secciones presenta enlucido de este tipo de material. Finalmente se sugiere que el Ministerio de Cultura exija a las instituciones estatales, al Gobierno Regional y a la Municipalidad Provincial de Vilcashuamán, que previa al inicio de cualquier tipo de obras de ingeniería e Infraestructura que se ejecuten dentro del perímetro de la actual ciudad de Vilcashuamán, se debe efectúe el respectivo Monitoreo Arqueológico a fin de registrar y preservar el Patrimonio de la Nación.

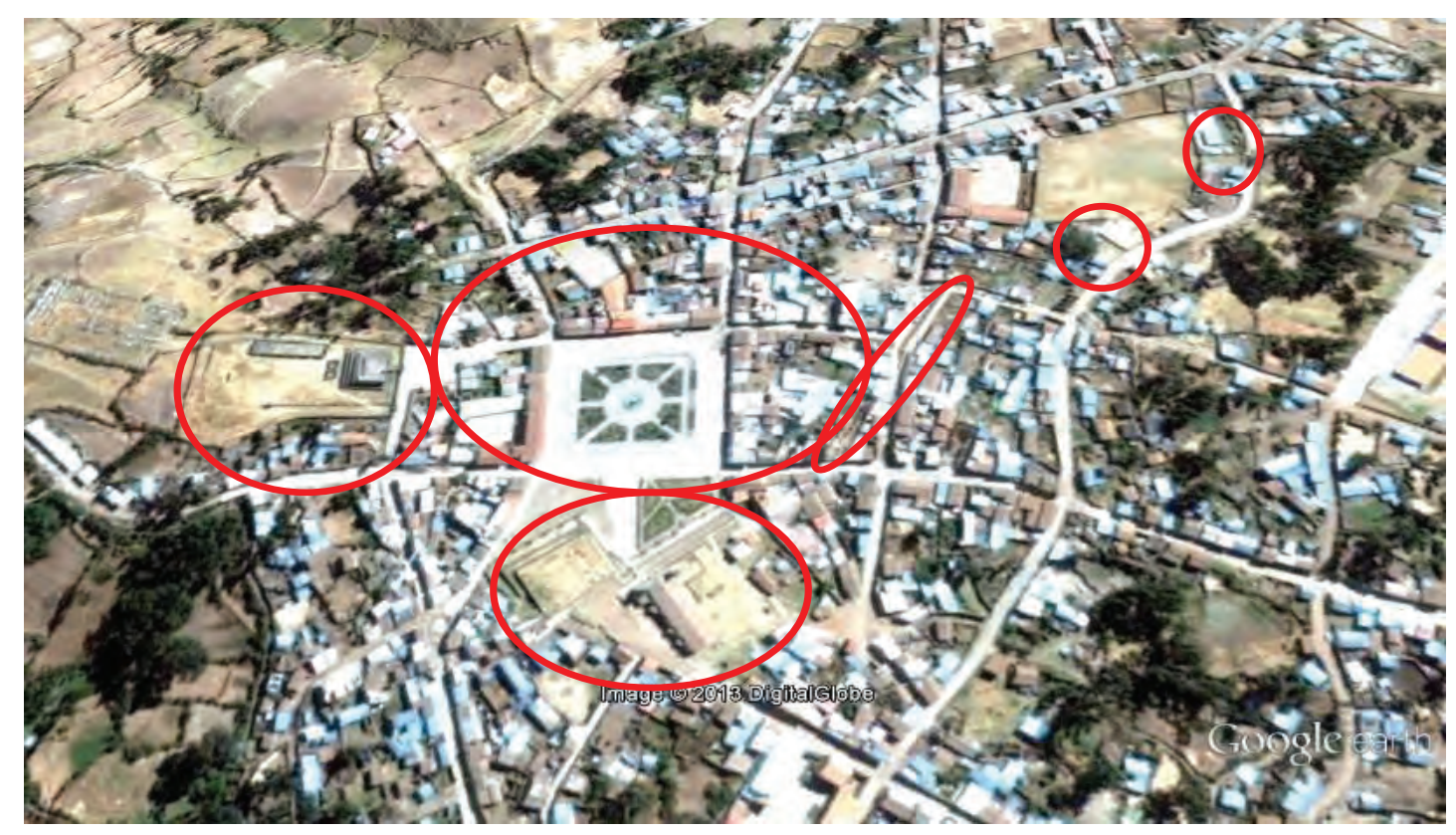

Figura 19: Vista satelital de la ciudad de Vilcashuamán y los sectores con evidencias arqueológicas actualmente visibles (Fuente: www.google earth). 


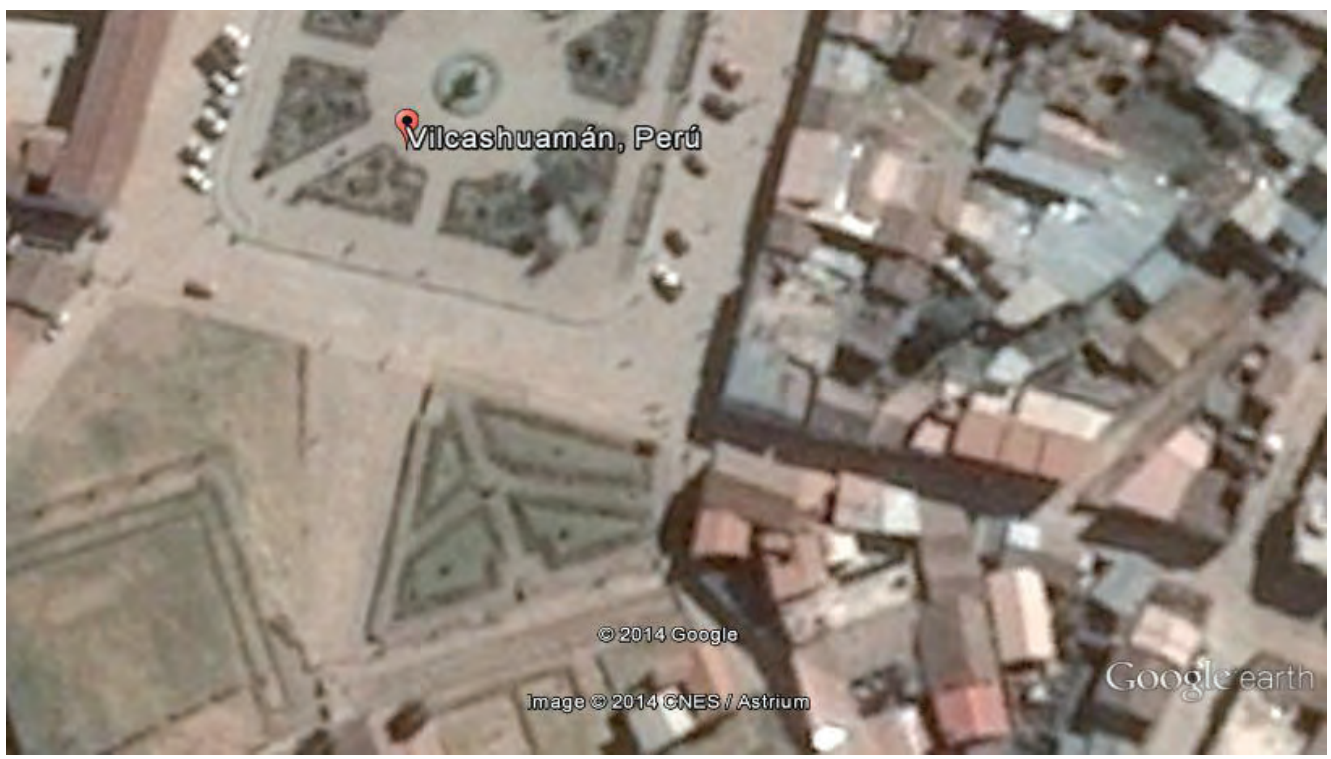

Figura 20: Vista satelital de la plaza de Vilcashuamán (1) y áreas colindantes; 2: Templo del Sol y la Luna; 3: Muro Pachacuteq; 4: Proyección de la dirección de desplazamiento del Canal-1 (Gentil acequia?); 5: Proyección de la dirección de desplazamiento del canal-2; 6 y 7: ubicación de los restos de calzada empedrada del Qhapaq Ñan.

(Modificado de www.googleearth.com)

\section{BiBLIOGRAFÍA}

CAVERO PALOMINO, Yuri.

2012 Informe Final del Monitoreo Arqueológico del proyecto: "Rehabilitación de pistas y veredas del cercado de Vilcashuamán, Provincia de Vilcashuamán - Ayacucho” (Código Snip 180804)

CIEZA DE LEÓN, Pedro

1967 El señorío de los Incas. Instituto de Estudios Peruanos. Lima.

COBO, Bernabé

1980 Historia del Nuevo Mundo (1653). Ediciones Atlas, Madrid.

GONZALES CARRE, Enrique; COSMOPOLIS, Jorge y Jorge LEVANO

1981 La ciudad inca de Vilcashuamán. Universidad San Cristóbal de Huamanga, Ayacucho

GUZMAN LADRON DE GUEVARA, Carlos

1959 Algunos Establecimientos Incas en la sierra central, Hoyas del Mantaro y del Pampas. En Actas y Trabajos del III Congreso Nacional de Historia del Perú, Vol 1. Edición Centro de Estudios Históricos Militares del Perú, Lima.

LUMBREAS SALCEDO, Luís Guillermo

1974 Las Fundaciones de Huamanga. Ediciones Nueva educación, Lima.

PEREZ, Ismael, PURIZAGA, Medardo y León, Freddy

2007 Vilcashuamán: Paisaje, Historia y Tradición. Universidad Nacional de San Cristóbal de Huamanga. Huamanga.

MATSUMO, Yuichi y Yuri CAVERO

2008 Campanayuq Rumi: Un centro ceremonial en forma de U en Vilcashuamán, Ayacucho, sierra centro sur. Resumen de ponencia presentado al VI Simposium Internacional de Arqueología "El Periodo Formativo: Enfoques y Evidencias Recientes, Cincuenta Años de la Misión Arqueológica Japonesa y su Vigencia. Organizado por la Pontificia Universidad Católica del Perú. Lima. 
SACO, Marcelo

2004 Componente Arqueológico. En: Proyecto Qhapaq Ñan. Informe de campaña 2004. Instituto Nacional de Cultura, Lima.

TORRES PALOMINO, Jorge

1969 Investigaciones Arqueológicas en Pillucho y Vilcashuamán. Instituto de Antropología, Universidad de San Cristóbal de Huamanga, Ayacucho.

VALDEZ, Lidio; VIVANCO, Cirilo y Casimiro CHAVEZ

1990 Asentamientos Chanka en la cuenca del Pampas y Qaracha. En Gaceta Arqueológica Andina, N 17, Lima. 
